\title{
No case of exercise-associated hyponatraemia in top male ultra-endurance cyclists: the 'Swiss Cycling Marathon'
}

\author{
Rüst, C A ; Knechtle, B ; Knechtle, P ; Rosemann, T
}

\begin{abstract}
The prevalence of exercise-associated hyponatraemia (EAH) has been investigated in endurance athletes such as runners and Ironman triathletes, but not in ultra-endurance road cyclists. We assessed fluid intake and changes in body mass, urine specific gravity and plasma sodium concentration $([\mathrm{Na}(+)])$ in 65 ultra-endurance road cyclists in a 720-km ultra-cycling marathon, the 'Swiss Cycling Marathon'. The cyclists lost $1.5(1.7) \%$ body mass $(\mathrm{P}<0.01)$. No athlete developed EAH. Fluid intake was associated with the change in plasma $[\mathrm{Na}(+)](\mathrm{r}=-0.32, \mathrm{P}<0.05)$ and the change in body mass $(\mathrm{r}=-0.30, \mathrm{P}<$ $0.05)$. The change in plasma $[\mathrm{Na}(+)]$ was related to post-race plasma $[\mathrm{Na}(+)](\mathrm{r}=0.63, \mathrm{P}<0.0001)$. To conclude, ad libitum fluid intake in ultra-endurance cyclists in a single-stage ultra-endurance road cycling race showed no case of EAH. Future studies regarding drinking behaviour in different ultra-endurance disciplines might give insights into why the prevalence of EAH is different in the different disciplines.
\end{abstract}

DOI: https://doi.org/10.1007/s00421-011-2024-y

Posted at the Zurich Open Repository and Archive, University of Zurich

ZORA URL: https://doi.org/10.5167/uzh-49166

Journal Article

Published Version

Originally published at:

Rüst, C A; Knechtle, B; Knechtle, P; Rosemann, T (2012). No case of exercise-associated hyponatraemia in top male ultra-endurance cyclists: the 'Swiss Cycling Marathon'. European Journal of Applied Physiology, 112(2):689-697.

DOI: https://doi.org/10.1007/s00421-011-2024-y 


\title{
No case of exercise-associated hyponatraemia in top male ultra-endurance cyclists: the 'Swiss Cycling Marathon'
}

\author{
Christoph Alexander Rüst • Beat Knechtle • \\ Patrizia Knechtle • Thomas Rosemann
}

Received: 22 February 2011 / Accepted: 23 May 2011/Published online: 9 June 2011

(c) Springer-Verlag 2011

\begin{abstract}
The prevalence of exercise-associated hyponatraemia $(\mathrm{EAH})$ has been investigated in endurance athletes such as runners and Ironman triathletes, but not in ultraendurance road cyclists. We assessed fluid intake and changes in body mass, urine specific gravity and plasma sodium concentration $\left(\left[\mathrm{Na}^{+}\right]\right)$in 65 ultra-endurance road cyclists in a 720-km ultra-cycling marathon, the 'Swiss Cycling Marathon'. The cyclists lost 1.5 (1.7)\% body mass $(P<0.01)$. No athlete developed EAH. Fluid intake was associated with the change in plasma $\left[\mathrm{Na}^{+}\right](r=-0.32$, $P<0.05)$ and the change in body mass $(r=-0.30$, $P<0.05)$. The change in plasma $\left[\mathrm{Na}^{+}\right]$was related to post-race plasma $\left[\mathrm{Na}^{+}\right](r=0.63, P<0.0001)$. To conclude, ad libitum fluid intake in ultra-endurance cyclists in a single-stage ultra-endurance road cycling race showed no case of EAH. Future studies regarding drinking behaviour in different ultra-endurance disciplines might give insights into why the prevalence of EAH is different in the different disciplines.
\end{abstract}

Keywords Hydration status · Body mass · Plasma sodium $\cdot$ Dehydration

Communicated by Susan A. Ward.

C. A. Rüst · B. Knechtle · P. Knechtle · T. Rosemann Institute of General Practice and for Health Services Research, University of Zurich, Zurich, Switzerland

B. Knechtle $(\square)$

Facharzt FMH für Allgemeinmedizin, Gesundheitszentrum St. Gallen, Vadianstrasse 26, 9001 St. Gallen, Switzerland e-mail: beat.knechtle@hispeed.ch

\section{Introduction}

Exercise-associated hyponatraemia (EAH) is a well-known and a well-investigated fluid and electrolyte disorder in endurance athletes and results from fluid retention in subjects who ingest abnormally large fluid volumes beyond the dictates of thirst during prolonged endurance exercise (Irving et al. 1991; Noakes et al. 1985, 2005; Speedy et al. 2001b). EAH was first described in the scientific literature in 1985 by Noakes et al. (1985) as being due to 'water intoxication'. The International Exercise-Associated Hyponatraemia Consensus Development Conference defined EAH as hyponatraemia occurring during or up to $24 \mathrm{~h}$ after prolonged physical activity (Hew-Butler et al. 2005, 2008).

The prevalence of EAH has been extensively investigated in endurance athletes such as marathoners (Almond et al. 2005; Chorley et al. 2007; Hew et al. 2003; Kipps et al. 2011; Mettler et al. 2008), ultra-marathoners (Knechtle et al. 2010a, 2011b; Lee et al. 2011; Reid and King 2007; Stuempfle et al. 2002, 2003) and Ironman triathletes (Hew-Butler et al. 2007; Sharwood et al. 2002; Speedy et al. 2001a). In marathoners, the prevalence of EAH amounts up to about $22 \%$ depending upon the number of investigated athletes, their gender and fitness level (Almond et al. 2005; Chorley et al. 2007; Hew et al. 2003; Kipps et al. 2011; Mettler et al. 2008). In ultra-marathoners, the prevalence of EAH varied between 0 and $44 \%$ (Knechtle et al. 2010a, 2011b; Reid and King 2007; Stuempfle et al. 2002, 2003). For Ironman triathletes, the prevalence of EAH was described in 1.8-28\% of the athletes (Speedy et al. 1997, 1999, 2001a; Wharam et al. 2006).

Regarding other endurance disciplines such as cycling, the literature on the prevalence of EAH is rather scarce. Two recent studies investigated fluid intake and electrolyte 
metabolism in ultra-endurance mountain bikers in multistage races (Rose and Peters 2010; Schenk et al. 2010). In the 3-day race of Rose and Peters (2010), the cyclists completed daily distances between 71 and $90 \mathrm{~km}$, respectively. In the 8-day race of Schenk et al. (2010), the cyclists had to complete daily distances between 62 and $122 \mathrm{~km}$, respectively. While in the 3 -day race over $248 \mathrm{~km}$, no change in altitude was indicated (Rose and Peters 2010), the athletes in the 8-day race had to climb a total of $21,691 \mathrm{~m}$ of altitude over the $665.4 \mathrm{~km}$ with $3,930 \mathrm{~m}$ of altitude being the highest altitude to climb in the steepest stage (Schenk et al. 2010). In both multi-stage races, no case of EAH was documented (Rose and Peters 2010; Schenk et al. 2010). Also, in a single-stage mountain bike ultra-marathon where athletes had to cover a total distance of $120 \mathrm{~km}$ with a total climb of $\sim 5,000 \mathrm{~m}$ in altitude, no case of EAH was reported (Knechtle et al. 2011c).

Literature regarding EAH in road cyclists is rather small. In one study on cyclists participating in a $109-\mathrm{km}$ cycle race, fluid metabolism was investigated (Hew-Butler et al. 2010). Out of the 33 investigated cyclists, 4 (12\%) developed EAH. In another study on 196 road cyclists in a 109-km cycling race, $1(0.5 \%)$ female athlete developed EAH. The athlete developed hyponatremic encephalopathy despite a modest fluid intake of $0.73 \mathrm{~L} / \mathrm{h}$ (Dugas and Noakes 2005). In a laboratory trial on 13 male and 14 female subjects, 1 female (7\%) became hyponatremic with symptoms during a water trial (Baker et al. 2005).

To date, no study investigated the prevalence of EAH in ultra-endurance road cyclists. For runners, a slow running pace and an excessive drinking behaviour (Hew-Butler et al. 2005, 2008) with a high frequency of fluid consumption (Almond et al. 2005) are considered as the main risk factors for fluid overload and subsequently developing EAH. In contrast to runners, cyclists are able to carry along up to four drinking bottles on their bikes and they can wear a backpack-type hydration pack on the back containing several litres of fluids with them while riding (Knechtle et al. 2011c). Based on this knowledge, we expected that the prevalence of EAH would be higher in ultra-endurance road cyclists compared to existing reports on marathoners.

The aim of the present study was therefore to explore the prevalence of EAH in male ultra-endurance road cyclists competing in the 'Swiss Cycling Marathon', a single-stage ultra-endurance cycling race. This race offers ultra-endurance cyclists to qualify for the 'Race across America', the 'RAAM' (Knechtle et al. 2011d). Athletes intending to qualify for the 'RAAM' have to qualify in an ultra-cycling race over $720 \mathrm{~km}$ (444 miles) and must finish within $115 \%$ of the winners' time in order to qualify. We hypothesised that the prevalence of EAH would be higher in these ultra-endurance cyclists compared to existing reports on marathoners. In case of an excessive fluid intake
(Hew-Butler et al. 2008; Speedy et al. 1997, 2001a), body mass would increase (Noakes et al. 2005; Speedy et al. 1997) and plasma $\left[\mathrm{Na}^{+}\right]$would decrease (Noakes et al. 2005; Speedy et al. 1997, 2001a).

\section{Materials and methods}

\section{Subjects}

The 'Swiss Cycling Marathon' (www.radmarathon.ch) takes place every year and offers a qualifier race to qualify for the 'Race Across America' (www.raceacrossamerica.org). To increase the sample size, data were collected during three consecutive years from 2008 to 2010 . The organiser of the 'Swiss Cycling Marathon' contacted all the race participants 3 months before the start of the race and provided information about the planned investigation. Interested athletes contacted the investigator by e-mail and were provided with study documentation. The study subjects were given information about the experimental procedure and they gave their informed written consent. Subjects were included in the study upon their first participation in the race; no athlete was included two or three times as study subject. The subjects were not given recommendations for their nutrition strategy, by the investigator or the race director (www.radmarathon. ch). The study was approved by the Institutional Review Board for the Use of Human Subjects of the Canton of St. Gallen, Switzerland. Sixty-five subjects of (mean and SD) 41.7 (7.1) years of age, 77.6 (7.9) $\mathrm{kg}$ of body mass, 1.80 (0.06) $\mathrm{m}$ of body height and a body mass index of $23.6(1.7)$ $\mathrm{kg} / \mathrm{m}^{2}$ finished the race.

\section{The race}

The 'Swiss Cycling Marathon' takes place at the end of June/start of July. In the $720-\mathrm{km}$ race, the athletes must first complete the $600-\mathrm{km}$ course of the race and then have to add an additional loop of $120 \mathrm{~km}$. In total, they have 11 checkpoints and $\sim 5,580 \mathrm{~m}$ of altitude to climb. The organiser offered at each checkpoint nutrition and fluids such as hypotonic sports drinks, soup, sandwiches, fruits, energy bars and muesli. The 600-km loop starts from the outskirts of Berne (Switzerland) leading over the border to Germany, then along Lake Constance into the Alps of Eastern Switzerland and back to Berne. After passing the transition area, the athletes complete the $120-\mathrm{km}$ loop in the western part of Switzerland. Weather conditions were comparable in all 3 years. Daily highs varied between $22^{\circ} \mathrm{C}$ and $25^{\circ} \mathrm{C}$, night lows were between $9^{\circ} \mathrm{C}$ and $11^{\circ} \mathrm{C}$.

Athletes intending to qualify for the 'RAAM' need to finish the $720 \mathrm{~km}$ within $115 \%$ of the winner's time, in 
general within 28:30 h:min for males younger than 50 years. In 2008, 23 athletes intended to qualify for the 'RAAM' where 5 athletes were able to qualify (22\%). In 2009, $34(60 \%)$ out of 56 'RAAM' qualifiers finished within the time limit and, in 2010, 11 (34\%) of 32 cyclists were able to qualify. In total, 50 cyclists $(45 \%)$ intending to qualify for the 'RAAM' were able to finish within the time limit in these 3 years. Apart from cyclists participating in the 'Swiss Cycling Marathon' to qualify for the 'RAAM', there were athletes competing in the $720-\mathrm{km}$ race without the intention of qualifying for the 'RAAM'. These athletes were also included as study participants. In 2008, 2 out of 8 $(25 \%)$ cyclists, in 2009, 14 out of $21(66 \%)$ and in 2010,6 out of $31(19 \%)$ athletes finished within the time limit.

\section{Measurements and calculations}

During the 3 months before the race, the participants kept a comprehensive training diary and recorded their cycling training sessions with distance, time and speed. The subjects were not asked about their experience in ultraendurance cycling, especially in participating in this specific race. Furthermore, the subjects were not asked about their knowledge on recommendations for fluid intake during ultra-endurance events. Before the start of the race, and immediately after arrival at the finish line, every participant underwent determination of anthropometric measurements such as body mass, thicknesses of the four skin folds (midupper arm, abdominal, mid-thigh, and mid-calf) and limb circumferences. Also, collecting of blood and urine samples was completed to measure haematocrit, plasma volume, plasma $\left[\mathrm{Na}^{+}\right]$and urine specific gravity. With the anthropometric data, body mass index, fat mass and skeletal muscle mass, using an anthropometric method, were estimated. The participants were weighed in their cycling dress without shoes or other equipment, after voiding their urinary bladder. Body mass was measured using a commercial scale (Beurer BF 15, Beurer, Ulm, Germany) to the nearest $0.1 \mathrm{~kg}$. Body height was determined using a stadiometer to the nearest $1.0 \mathrm{~cm}$. The circumferences of the limbs were measured on the right side of the body, using a non-elastic tape measure $(\mathrm{cm})$ (KaWe CE, Kirchner und Welhelm, Germany) to the nearest $0.1 \mathrm{~cm}$. The circumference of the upper arm was measured at mid-upper arm, the circumference of the thigh was taken at mid-thigh and the circumference of the calf was measured at mid-calf. The skin fold data were obtained using a skin fold calliper (GPM-Hautfaltenmessgerät, Siber \& Hegner, Zurich, Switzerland) on the right side of the body and recorded to the nearest $0.2 \mathrm{~mm}$. The skin fold measurements were taken following ISAK standard once for all skin folds and then the procedure was repeated twice more by the same investigator; the mean of the three measurements was then used for the analyses. The timing of the taking of the skin fold measurements was standardised to ensure reliability. According to Becque et al. (1986), readings were performed $4 \mathrm{~s}$ after applying the calliper. One trained investigator took all the skin fold measurements as inter-tester variability is a major source of error in skin fold measurements. An intra-tester reliability check was conducted on 27 male athletes prior to testing. Intra-class correlation (ICC) within the two judges was excellent for all anatomical measurement sites and various summary measurements of skin fold thicknesses (ICC > 0.9). Agreement tended to be higher within measurers than between measurers, but still reached excellent reliability $(\mathrm{ICC}>0.9)$ for the summary measurements of skin fold thicknesses (Knechtle et al. 2010b). Fat mass was estimated using the equations from Stewart and Hannan (2000) for male athletes: fat mass $(\mathrm{g})=331.5 \times$ (abdominal skin fold thickness $)+356.2 \times($ thigh skin fold thickness $)+111.9 \times$ (body mass) - 9,108. Skeletal muscle mass (kg) was estimated using the anthropometric equation of Lee et al. (2000) with skeletal muscle mass $=\mathrm{Ht} \times(0.00744 \times$ $\left.\mathrm{CAG}^{2}+0.00088 \times \mathrm{CTG}^{2}+0.00441 \times \mathrm{CCG}^{2}\right)+2.4 \times$ sex $-0.048 \times$ age + race +7.8 where $\mathrm{Ht}=$ height, $\mathrm{CAG}=$ skin fold-corrected upper arm girth, $\mathrm{CTG}=$ skin fold-corrected thigh girth, $\mathrm{CCG}=$ skin fold-corrected calf girth, $\operatorname{sex}=1$ for male; age is in years; race $=0$ for white men and 1 for black men. After weighing, capillary blood samples of $80 \mu \mathrm{l}$ were drawn from a fingertip and both haematocrit and plasma sodium concentration $\left(\left[\mathrm{Na}^{+}\right]\right)$ were analysed using the i-STAT ${ }^{\circledR} 1$ System (Abbott Laboratories, Abbott Park, IL, USA). Changes in plasma volume were calculated from the pre- and post-race haematocrit values according to Beaumont (1972). Urine specific gravity was analysed using Clinitek Atlas ${ }^{\circledR}$ Automated Urine Chemistry Analyzer (Siemens Healthcare Diagnostics, Deerfield, IL, USA). During the race, the participants recorded their fluid intake. At each aid station, the participants wrote down the number of cups or drink bottles they consumed and the total fluid intake was calculated. Fluid intake immediately before the start and after the finish was added to the fluid intake during the race. Upon arrival to the investigators post-race, participants were asked for symptoms of EAH (Hew-Butler et al. 2005, 2008).

\section{Statistical analysis}

Results are presented as mean and standard deviation (SD). The paired $t$ test was used to check for significant changes in the parameters during the race and Pearson correlation analysis was applied to check for associations between the parameters. For all statistical tests, significance was set at $P<0.05$. 


\section{Results}

During training, the participants completed 340 (116) cycling kilometres per week within 13.1 (4.7) h riding at a mean speed of $26.8(5.7) \mathrm{km} / \mathrm{h}$. While racing, the participants rode at a mean speed of $25.2(3.5) \mathrm{km} / \mathrm{h}$ and completed the $720 \mathrm{~km}$ within 1,733 (261) min. Speed during the race was significantly slower compared to the speed during training $(P<0.05)$.

Plasma $\left[\mathrm{Na}^{+}\right]$remained stable and no participant developed EAH (see Table 1). The participants consumed on average $0.67(0.23) \mathrm{L} / \mathrm{h}$, equal to 8.8 (3.2) $\mathrm{mL} \mathrm{kg}^{-1} \mathrm{~h}^{-1}$. Fluid intake showed no correlation with race time, post-race plasma $\left[\mathrm{Na}^{+}\right]$or post-race body mass $(P>0.05)$. Fluid intake was significantly and negatively associated with the change in plasma $\left[\mathrm{Na}^{+}\right](r=-0.30, P=0.01)$ (see Fig. 1) and the change in body mass $(r=-0.28, P=0.02)$ (see Fig. 2). While looking at the data distribution in Fig. 2, one can notice that the correlation between fluid intake and change in body mass was highly affected by two participants who drank $\sim 0.7 \mathrm{~L} / \mathrm{h}$ and $\sim 1.3 \mathrm{~L} / \mathrm{h}$, respectively. Without the data of these two participants (who could have drank much, but possibly eaten only a little or not at all during the race), the correlation became insignificant with $r=-0.15$ $(P>0.05)$. The change in plasma $\left[\mathrm{Na}^{+}\right]$was significantly and positively related to post-race plasma $\left[\mathrm{Na}^{+}\right](r=0.63$, $P<0.0001)$.

The participants lost $1.2(1.3) \mathrm{kg}$ in body mass, equal to 1.5 (1.7)\% body mass $(P<0.01)$ (see Table 1$)$. The change in body mass varied between $-10 \%$ and $+4 \%$ (see Fig. 3) and was significantly and negatively related to overall race time $(r=-0.62, P<0.0001)$ (see Fig. 4). However, the change in body mass was neither related to post-race plasma $\left[\mathrm{Na}^{+}\right](r=0.06, P=0.61)$ (see Fig. 5) nor to the change in plasma $\left[\mathrm{Na}^{+}\right](r=0.19, P=0.13)$ (see Fig. 6). Skeletal muscle mass remained unchanged and fat mass decreased highly significantly $(P<0.01)$. The decrease in fat mass was not related to the decrease in body mass $(P>0.05)$. Plasma volume increased by 0.32 (10.9)\% $(P>0.05)$ and urine specific gravity increased significantly by $0.6(0.9) \%(P<0.05)$. The change in body

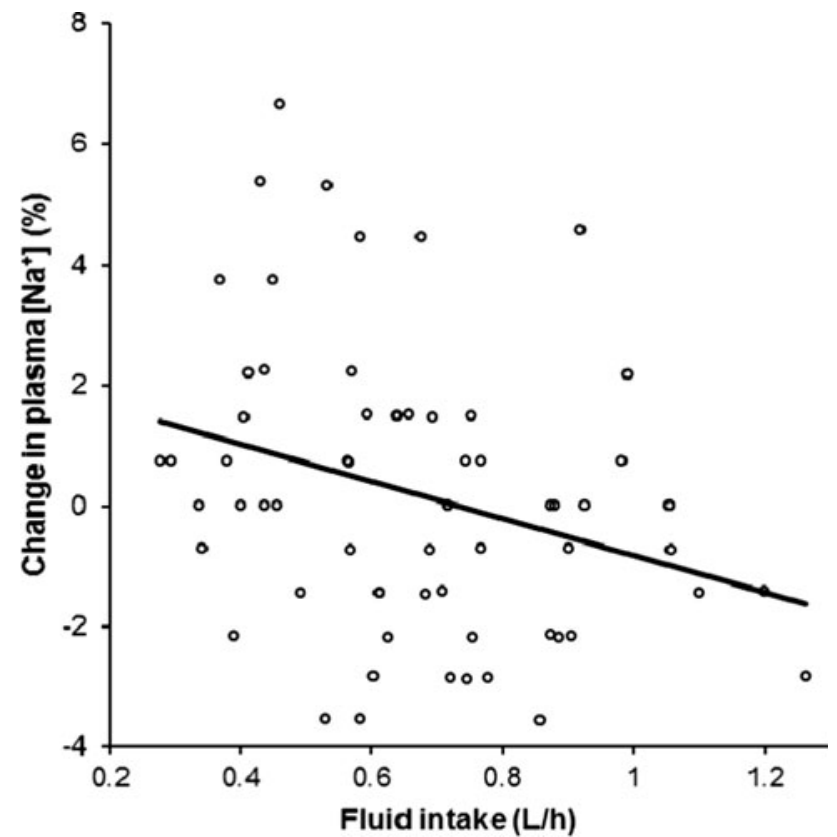

Fig. 1 The relationship between fluid intake and change in plasma $\left[\mathrm{Na}^{+}\right]$

mass showed no association to post-race plasma $\left[\mathrm{Na}^{+}\right]$, the change in plasma $\left[\mathrm{Na}^{+}\right]$or race time $(P>0.05)$.

\section{Discussion}

The aim of this study was to explore the prevalence of EAH in male ultra-endurance road cyclists in the 'Swiss Cycling Marathon', a single-stage ultra-cycling race and a qualifier for the 'RAAM'. We hypothesised that the prevalence of EAH would be higher compared to reports on marathoners. However, no athlete developed EAH.

Fluid intake and prevalence of exercise-associated hyponatraemia

The main finding in this study was that no ultra-endurance cyclist in the 'Swiss Cycling Marathon' developed EAH.

Table 1 Mean values and standard deviation (SD) of body mass and laboratory parameters before and after the race for the 65 athletes

\begin{tabular}{llccc}
\hline & Pre-race & Post-race & Absolute change & Change in \% \\
\hline Body mass $(\mathrm{kg})$ & $77.6(7.9)$ & $76.4(7.8)$ & $-1.2(1.3)$ & $-1.5(1.7)^{* *}$ \\
Skeletal muscle mass $(\mathrm{kg})$ & $40.2(3.6)$ & $40.4(3.4)$ & $+0.2(1.2)$ & $+0.6(3.4)$ \\
Fat mass $(\mathrm{kg})$ & $9.0(4.2)$ & $7.4(3.7)$ & $-1.6(1.4)$ & $-19.5(12.5)^{* *}$ \\
Haematocrit $(\%)$ & $44.4(2.6)$ & $44.5(2.7)$ & $+0.06(2.6)$ & $+0.28(5.78)$ \\
Plasma $\left[\mathrm{Na}^{+}\right](\mathrm{mmol} / \mathrm{L})$ & $137.4(2.5)$ & $137.6(1.7)$ & $+0.18(3.28)$ & $+0.16(2.34)$ \\
Urine specific gravity $(\mathrm{g} / \mathrm{mL})$ & $1.013(0.0008)$ & $1.019(0.0009)$ & $+0.006(0.0009)$ & $+0.57(0.89)^{* *}$ \\
\hline
\end{tabular}

** $P<0.01$ 


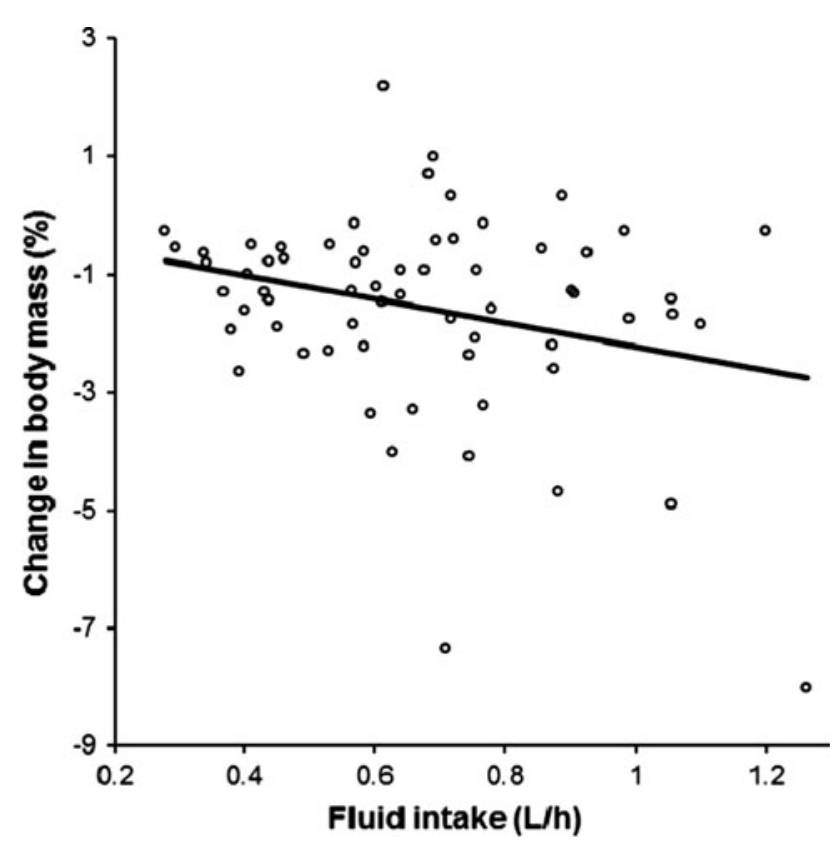

Fig. 2 The relationship between fluid intake and change in body mass

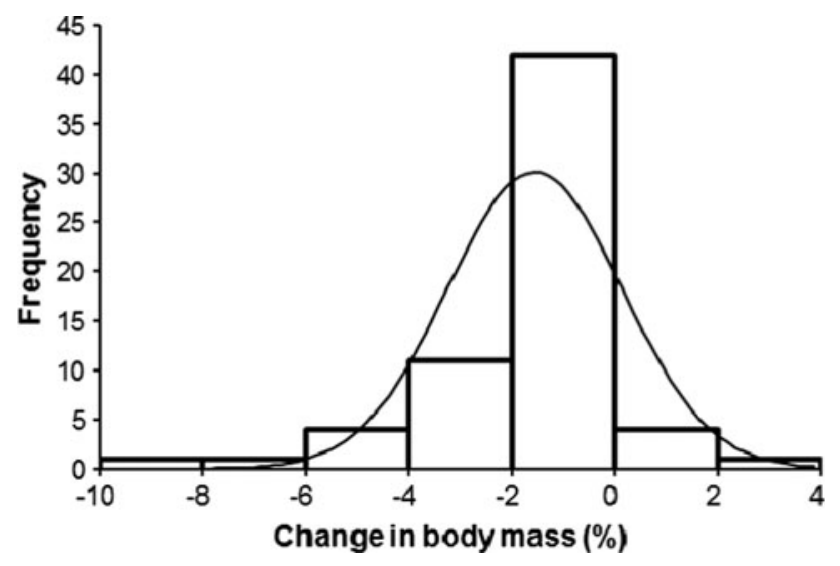

Fig. 3 The variation of change in body mass

EAH is a dilutional hyponatraemia caused by the intake of hypotonic fluids beyond the dictate of thirst (Hew-Butler et al. 2005, 2008; Irving et al. 1991). In marathoners, hyponatremic athletes reported significantly larger fluid intakes than non-hyponatremic finishers (Almond et al. 2005; Kipps et al. 2011). Hyponatremic marathoners consume fluids at a higher rate $(0.84 \mathrm{~L} / \mathrm{h})$ compared to non-hyponatremic runners $(0.45 \mathrm{~L} / \mathrm{h})$ (Kipps et al. 2011). Furthermore, fluid intake correlated significantly with plasma $\left[\mathrm{Na}^{+}\right]$change between the start and the finish of the race in marathoners (Mettler et al. 2008).

In these ultra-endurance cyclists, fluid intake was relatively high with $0.67(0.23) \mathrm{L} / \mathrm{h}$ compared to marathoners (Kipps et al. 2011) and no athlete developed EAH.

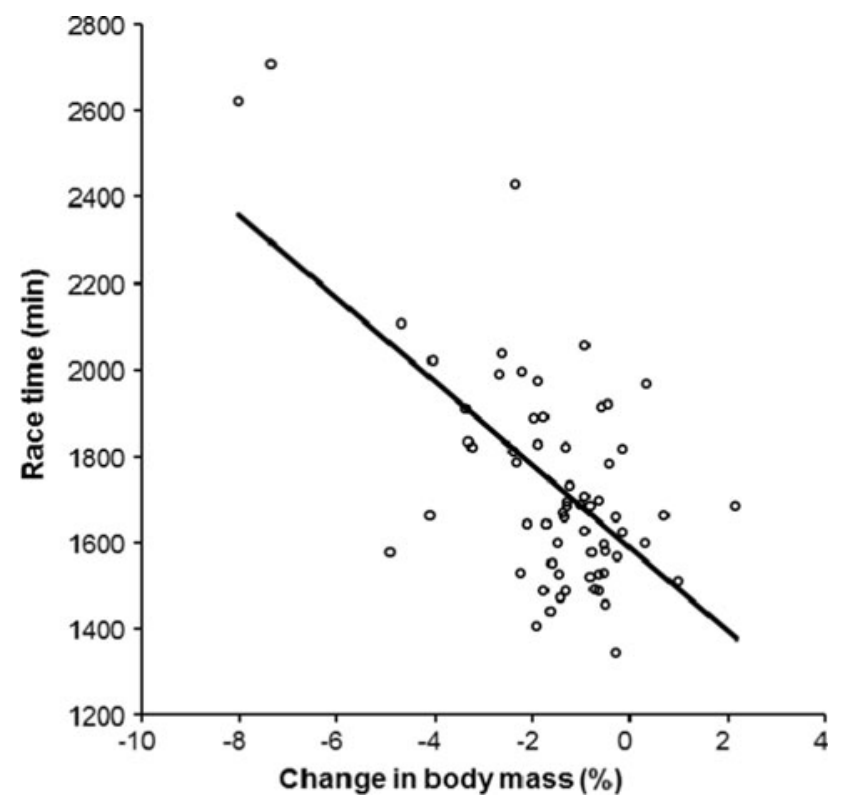

Fig. 4 The relationship between change in body mass and race time

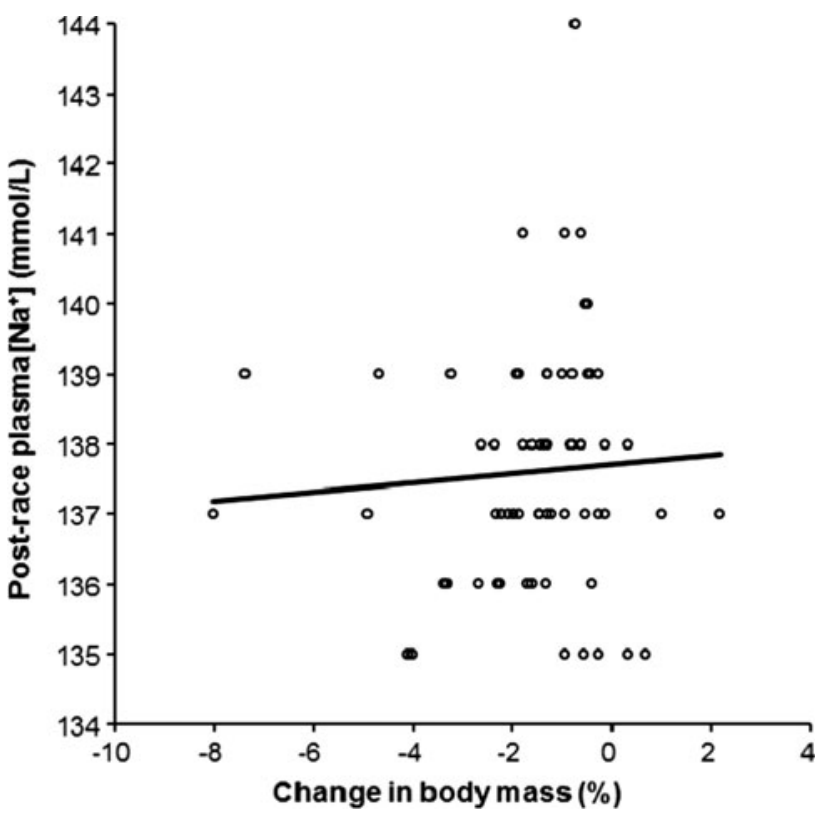

Fig. 5 The association between the change in body mass and postrace plasma $\left[\mathrm{Na}^{+}\right]$

Consumption of this fluid volume was still within the hourly drinking volume limits of $0.8 \mathrm{~L} / \mathrm{h}$ recommended for endurance exercise (Noakes 2003; Sawka et al. 2007). Also, in the multi-stage mountain bike race of Schenk et al. (2010), fluid intake did not exceed $0.75 \mathrm{~L} / \mathrm{h}$. In the multistage mountain bike race of Rose and Peters (2010), fluid intake was even lower ranging between 0.34 and $0.55 \mathrm{~L} / \mathrm{h}$. Neither in our participants nor in the subjects in the multistage races (Rose and Peters 2010; Schenk et al. 2010), any case of EAH occurred in ultra-endurance cyclists. 


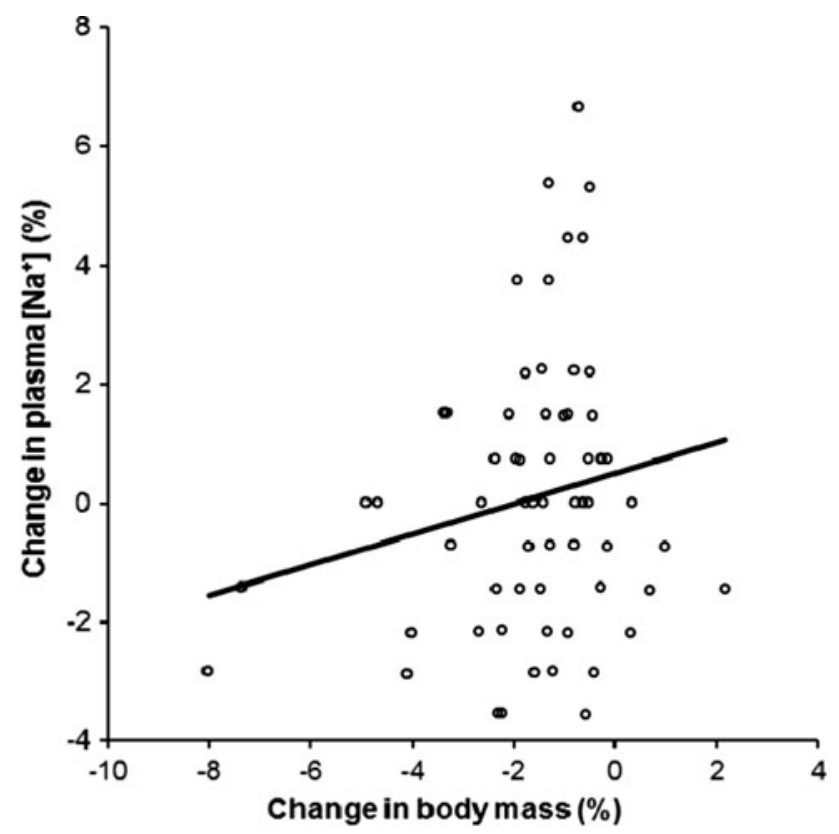

Fig. 6 The relationship between the change in body mass and the change in plasma $\left[\mathrm{Na}^{+}\right]$

While Schenk et al. (2010) described five and four cases, respectively, of asymptomatic hypernatremia, defined as plasma $\left[\mathrm{Na}^{+}\right]>145 \mathrm{mmol} / \mathrm{L}$, at the finish of Stages 5 and 6 in their multi-stage race, no subject in the present singlestage ultra-endurance cycling race developed hypernatremia. The highest post-race plasma $\left[\mathrm{Na}^{+}\right]$was $142 \mathrm{mmol} / \mathrm{L}$ in two athletes. Presumably, the rather higher fluid intake in our athletes prevented hypernatremia.

The common opinion about EAH is that the slower athletes drink more than faster ones (Hew-Butler et al. 2005, 2008) thus leading to fluid overload. In these ultraendurance cyclists, however, we found no case of EAH. The low fluid intake of our athletes, as well as the even lower fluid intake in the mentioned multi-stage mountain bikers, may be the reason that neither in the mentioned mountain bikers nor in the present ultra-endurance cyclists cases with EAH were found. Schenk et al. (2010) hypothesised that the high intensity, difficult terrain, impossibility of drafting and the descents were the main reasons that mountain bikers drink rather little compared to other ultra-endurance athletes such as Ironman triathletes (Speedy et al. 2001a).

Change in body mass and plasma sodium

A second hypothesis was that body mass would increase and plasma $\left[\mathrm{Na}^{+}\right]$decrease in case of excessive fluid intake. In case of dilutional hyponatraemia due to inappropriate fluid intake, an increase in body mass (Noakes et al. 2005; Speedy et al. 1997) and a decrease in plasma
$\left[\mathrm{Na}^{+}\right]$(Noakes et al. 2005; Speedy et al. 1997, 2001a, b) would be expected. In endurance athletes, an association between the change in body mass and both post-race plasma $\left[\mathrm{Na}^{+}\right]$and the change in plasma $\left[\mathrm{Na}^{+}\right]$has been described (Mettler et al. 2008; Sharwood et al. 2002). In marathoners developing EAH, the change in body mass correlated with both post-race plasma $\left[\mathrm{Na}^{+}\right]$and the change in plasma $\left[\mathrm{Na}^{+}\right]$(Mettler et al. 2008).

Noakes et al. (2005) investigated 2,135 athletes and could clearly demonstrate that weight gain consequent to excessive fluid consumption was the principal cause of a reduced serum $\left[\mathrm{Na}^{+}\right]$after exercise. In these ultra-endurance cyclists, fluid intake was significantly and negatively related to both the change in plasma $\left[\mathrm{Na}^{+}\right]$and the change in body mass. Athletes with an increased fluid intake showed a decrease in both plasma $\left[\mathrm{Na}^{+}\right]$and body mass. While plasma $\left[\mathrm{Na}^{+}\right]$decreased with increased fluid intake in these ultra-endurance cyclists, body mass also decreased.

However, it is difficult to explain the decrease in body mass despite the increased fluid intake. Presumably the decrease in body mass was due to dehydration since urine specific gravity increased (Kavouras 2002). The change in body mass was neither related to post-race plasma $\left[\mathrm{Na}^{+}\right]$ nor to the change in plasma $\left[\mathrm{Na}^{+}\right]$. The decrease in body mass and the increase in urine specific gravity indicated dehydration (Kavouras 2002; Shireffs 2003), rather than hyperhydration. However, body weight changes under the testing conditions do not reflect a change in body hydration, but rather represent a balance (combined effect) of both fluid and energy (in food and drinks) intake and both fluid and energy losses from both external and internal sources (significant fat mass losses) during the race. In this case, urine specific gravity might be considered as a better indicator of body hydration. However, it must be acknowledged that its reliability under the testing conditions is also limited.

Mettler et al. (2008) showed in marathoners an association between the change in body mass and the change in both post-race plasma $\left[\mathrm{Na}^{+}\right]$and the change in plasma $\left[\mathrm{Na}^{+}\right]$, and an association between post-race plasma $\left[\mathrm{Na}^{+}\right]$ and the change in plasma $\left[\mathrm{Na}^{+}\right]$. We could, however, not confirm their first finding, but were able to replicate their second finding. The decrease in body mass might, however, also be due to a decrease in solid masses as has been found in ultra-runners (Knechtle et al. 2011a) and ultra-cyclists (Knechtle et al. 2009a, b) where fat mass (Knechtle et al. 2009b, 2011a) and muscle mass (Knechtle et al. 2009a) decreased in ultra-endurance athletes. Although fat mass decreased in these ultra-endurance cyclists, the decrease was not associated with a decrease in body mass.

Fluid intake showed no association with post-race body mass, however, with the change in body mass (see Fig. 2). 
In case of fluid overload with development of EAH, we would have expected an increase in body mass as has been found in marathon runners (Almond et al. 2005; Kipps et al. 2011). Body mass, however, decreased in these athletes by $1.5 \%$. The mean fluid intake of $0.67(0.23) \mathrm{L} / \mathrm{h}$ prevented obviously from weight gain and consequently from the development of EAH (Hew-Butler et al. 2005, 2008). Following the 'Position Stand' of the American College of Sports Medicine for 'Exercise and Fluid Replacement' (Sawka et al. 2007), a fluid intake between 0.4 and $0.8 \mathrm{~L} / \mathrm{h}$ is recommended for marathoners. Obviously, these ultra-cyclists met exactly these recommendations. The decrease in body mass and the increase in urine specific gravity indicated dehydration (Kavouras 2002; Shireffs 2003). However, the use of urine specific gravity as a marker of hydration status is time dependent and shows only chronic dehydration, but not acute dehydration (Sawka et al. 2007).

Lower prevalence of EAH in Switzerland compared to other countries?

The present investigation is another study to show that the prevalence of EAH is very low in both marathon runners and ultra-endurance athletes competing in Switzerland. In the 'Zurich Marathon', Mettler et al. (2008) showed a low prevalence of $3 \%$ of EAH. In male 24-h ultra-marathoners (Knechtle et al. 2010a) and in male ultra-endurance mountain bikers (Knechtle et al. 2011c), no case of EAH was found. For ultra-marathoners in a 100-km ultra-marathon, the prevalence of EAH was low at $\sim 5 \%$ (Knechtle et al. 2011b). These results are far below the reported prevalence rates of EAH in other studies in other countries as cited in "Introduction".

We assume that drinking behaviour among both ultraendurance athletes (Knechtle et al. 2010a, 2011b, c, e) and marathoners (Mettler et al. 2008) is different in Switzerland compared to other countries. Drinking policies during exercise have changed substantially throughout history. There has been an increase in the promotion of overdrinking by athletes since the mid-1990s (Beltrami et al. 2008). Runners have a poor understanding of the physiological consequences of hydration behaviours that frequently reflect messages of advertising. Athletes at the highest risk of EAH exhibit behaviour that is shaped by their beliefs about the benefits and risks of hydration. These beliefs are frequently based on misconceptions about basic exercise physiology (Winger et al. 2011). Presumably, these beliefs are triggered by the sports industry. Noakes (2011) reported in an actual review that overdrinking is linked to influential drinking guidelines.

Another aspect regarding hydration status might be the previous experience of the athletes. In a study on 42 participants in a Swiss mountain marathon over $7 \mathrm{~h} 3 \mathrm{~min}$, the subjects consumed $0.6 \mathrm{~L}$ of fluids per hour (Kruseman et al. 2005). No athlete was at risk for fluid overload; body composition and race experience correlated to race time. The authors assumed that experienced ultra-runners were well trained and knew their personal needs during such an ultra-endurance race.

\section{Limitations}

A limitation of this investigation is the fact that we were not able to record cups or bottles drunk in part only and the amount of fluid splashed while drinking. This could increase the error of the subjects self-reporting of the number of cups and bottles of fluid. A further limitation is that we did not determine the sodium content in the ingested fluids and food; sodium intake might be associated with post-race plasma $\left[\mathrm{Na}^{+}\right]$. The amount of sodium delivered to the body with the food could likely have a higher impact on post-race plasma $[\mathrm{Na}+]$ than sodium consumed in beverages, influencing the association between fluid intake and change in plasma $[\mathrm{Na}+]$. However, the content of sodium in neither one was assessed.

However, two studies with Ironman triathletes showed that sodium supplementation was not required to maintain plasma $\left[\mathrm{Na}^{+}\right]$(Hew-Butler et al. 2006) or to prevent EAH (Speedy et al. 2002). A further limitation was that we did not determine plasma $\left[\mathrm{Na}^{+}\right]$in the non-finishers. The dropout rate in the 'Swiss Cycling Marathon' in the RAAM qualifier is rather high (Knechtle et al. 2011d). The inclusion of non-finishers might have shown some cases of EAH. A further limitation was the non-recording of urine production. Recording the water loss via urine might help to explain the decrease in body mass despite the increased fluid intake.

To summarise, ad libitum fluid intake in ultra-endurance cyclists in a single-stage ultra-endurance road cycling race showed no case of EAH, as has already been described in both single-stage and multi-stage mountain bike ultramarathoners. Future studies regarding drinking behaviour in different ultra-endurance disciplines might give insights into why the prevalence of EAH is different in the different disciplines.

\section{References}

Almond CS, Shin AY, Fortescue EB, Mannix RC, Wypij D, Binstadt BA, Duncan CN, Duncan CN, Olson DP, Salerno AE, Newburger JW, Greenes DS (2005) Hyponatremia among runners in the Boston Marathon. N Engl J Med 352:1550-1556

Baker LB, Munce TA, Kenney WL (2005) Sex differences in voluntary fluid intake by older adults during exercise. Med Sci Sports Exerc 37:789-796 
Becque MD, Katch VL, Moffatt RJ (1986) Time course of skin-plusfat compression in males and females. Hum Biol 58:33-42

Beltrami FG, Hew-Butler T, Noakes TD (2008) Drinking policies and exercise-associated hyponatraemia: is anyone still promoting overdrinking? Br J Sports Med 42:496-501

Chorley J, Cianca J, Divine J (2007) Risk factors for exerciseassociated hyponatremia in non-elite marathon-runners. Clin J Sport Med 17:471-477

Dugas JP, Noakes TD (2005) Hyponatraemic encephalopathy despite a modest rate of fluid intake during a $109 \mathrm{~km}$ cycle race. Br J Sports Med 39:e38 discussion e38

Hew TD, Chorley JN, Cianca JC, Divine JG (2003) The incidence, risk factors and clinical manifestations of hyponatremia in marathon runners. Clin J Sport Med 13:41-47

Hew-Butler T, Almond C, Ayus JC, Dugas J, Meeuwisse W, Noakes T, Reid S, Siegel A, Speedy D, Stuempfle K, Verbalis J, Weschler L, Exercise-Associated Hyponatremia (EAH) Consensus Panel (2005) Consensus statement of the First International Exercise-Associated Hyponatremia Consensus Development Conference, Cape Town, South Africa. Clin J Sport Med 15:208-213

Hew-Butler TD, Sharwood K, Collins M, Speedy D, Noakes T (2006) Sodium supplementation is not required to maintain serum sodium concentrations during an Ironman triathlon. Br J Sports Med 40:255-259

Hew-Butler T, Collins M, Bosch A, Sharwood K, Wilson G, Armstrong M, Jennings C, Swart J, Noakes T (2007) Maintenance of plasma volume and serum sodium concentration despite body weight loss in ironman triathletes. Clin J Sport Med 17:116-122

Hew-Butler T, Ayus JC, Kipps C, Maughan RJ, Mettler S, Meeuwisse WH, Page AJ, Reid SA, Rehrer NJ, Roberts WO, Rogers IR, Rosner MH, Siegel AJ, Speedy DB, Stuempfle KJ, Verbalis JG, Weschler LB, Wharam P (2008) Statement of the Second International Exercise-Associated Hyponatremia Consensus Development Conference, New Zealand, 2007. Clin J Sport Med 18:111-121

Hew-Butler T, Dugas JP, Noakes TD, Verbalis JG (2010) Changes in plasma arginine vasopressin concentrations in cyclists participating in a 109-km cycle race. Br J Sports Med 44:594-597

Irving RA, Noakes TD, Buck R, van Zyl Smit R, Raine E, Godlonton J, Norman RJ (1991) Evaluation of renal function and fluid homeostasis during recovery from exercise-induced hyponatremia. J Appl Physiol 70:342-348

Kavouras SA (2002) Assessing hydration status. Curr Opin Clin Nutr Metab Care 5:519-524

Kipps C, Sharma S, Pedoe DT (2011) The incidence of exerciseassociated hyponatremia in the London Marathon. Br J Sports Med 45:14-19

Knechtle B, Knechtle P, Rosemann T, Senn O (2009a) No dehydration in mountain bike ultra-marathoners. Clin J Sport Med 19:415-420

Knechtle B, Wirth A, Knechtle P, Rosemann T (2009b) An ultracycling race leads to no decrease in skeletal muscle mass. Int $\mathbf{J}$ Sports Med 30:163-167

Knechtle B, Joleska I, Wirth A, Knechtle P, Rosemann T, Senn O (2010a) Intra- and inter-judge reliabilities in measuring the skinfold thicknesses of ultra runners under field conditions. Percept Mot Skills 111:105-106

Knechtle B, Knechtle P, Rosemann T (2010b) No exercise-associated hyponatremia found in an observational field study of male ultramarathoners participating in a 24-hour ultra-run. Phys Sportsmed 38:94-100

Knechtle B, Wirth A, Knechtle P, Rosemann T, Senn O (2011a) Do ultra-runners in a $24-\mathrm{h}$ run really dehydrate? Ir J Med Sci 180:129-134
Knechtle B, Knechtle P, Rosemann T (2011b) Low prevalence of exercise-associated hyponatremia in male $100 \mathrm{~km}$ ultra-marathon runners in Switzerland. Eur J Appl Physiol 111(6):1007-1016

Knechtle B, Knechtle P, Rosemann T (2011c) No case of exerciseassociated hyponatremia in male ultra-endurance mountain bikers in the 'Swiss Bike Masters'. Chin J Physiol. doi: 10.4077/CJP.2011.AMM050

Knechtle B, Knechtle P, Rüst CA, Rosemann T, Lepers R (2011d) Finishers and non-finishers in the 'Swiss Cycling Marathon' to qualify for the 'Race across America'. J Strength Cond Res, in press

Knechtle B, Gnädinger M, Knechtle P, Imoberdorf R, Kohler G, Ballmer P, Rosemann T, Senn O (2011e) Prevalence of exerciseassociated hyponatremia in male ultraendurance athletes. Clin $\mathrm{J}$ Sport Med 21(3):226-232

Kruseman M, Bucher S, Bovard M, Kayser B, Bovier PA (2005) Nutrient intake and performance during a mountain marathon: an observational study. Eur J Appl Physiol 94:151-157

Lee RC, Wang Z, Heo M, Ross R, Janssen I, Heymsfield SB (2000) Total-body skeletal muscle mass: development and cross-validation of anthropometric prediction models. Am J Clin Nutr 72:796-803

Lee JK, Nio AQ, Ang WH, Johnson C, Aziz AR, Lim CL, HewButler T (2011) First reported cases of exercise-associated hyponatremia in Asia. Int J Sports Med, epub ahead of print

Mettler S, Rusch C, Frey WO, Bestmann L, Wenk C, Colombani PC (2008) Hyponatremia among runners in the Zurich Marathon. Clin J Sport Med 18:344-349

Noakes TD (2003) Overconsumption of fluids by athletes. BMJ 327:113-114

Noakes TD (2011) Changes in body mass alone explain almost all of the variance in the serum sodium concentrations during prolonged exercise. Has commercial influence impeded scientific endeavour? Br J Sports Med, epub ahead of print

Noakes TD, Goodwin N, Rayner BL, Branken T, Taylor RK (1985) Water intoxication: a possible complication during endurance exercise. Med Sci Sports Exerc 17:370-375

Noakes TD, Sharwood K, Speedy D, Hew T, Reid S, Dugas J, Almond C, Wharam P, Weschler L (2005) Three independent biological mechanisms cause exercise-associated hyponatremia: evidence from 2, 135 weighed competitive athletic performances. Proc Natl Acad Sci USA 102:18550-18555

Reid SA, King MJ (2007) Serum biochemistry and morbidity among runners presenting for medical care after an Australian mountain ultramarathon. Clin J Sport Med 17:307-310

Rose S, Peters-Futre EM (2010) Ad libitum adjustments to fluid intake in cool environmental conditions maintain hydration status in a three-day mountain bike race. Br J Sports Med 44:430-436

Sawka MN, Burke LM, Eichner ER, Maughan RJ, Montain SJ, Stachenfeld NS, American College of Sports Medicine (2007) American College of Sports Medicine position stand. Exercise and fluid replacement. Med Sci Sports Exerc 39:377-390

Schenk K, Gatterer H, Ferrari M, Ferrari P, Cascio VL, Burtscher M (2010) Bike Transalp 2008: liquid intake and its effect on the body's fluid homeostasis in the course of a multistage, crosscountry, MTB marathon race in the central Alps. Clin J Sport Med 20:47-52

Sharwood K, Collins M, Goedecke J, Wilson G, Noakes T (2002) Weight changes, sodium levels, and performance in the South African Ironman Triathlon. Clin J Sport Med 12:391-399

Shirreffs SM (2003) Markers of hydration status. Eur J Clin Nutr 57:S6-S9

Speedy DB, Faris JG, Hamlin M, Gallagher PG, Campbell RG (1997) Hyponatremia and weight changes in an ultradistance triathlon. Clin J Sport Med 7:180-184 
Speedy DB, Noakes TD, Rogers IR, Thompson JM, Campbell RG, Kuttner JA, Boswell DR, Wright S, Hamlin M (1999) Hyponatremia in ultradistance triathletes. Med Sci Sports Exerc 31:809-815

Speedy DB, Noakes TD, Kimber NE, Rogers IR, Thompson JM, Boswell DR, Ross JJ, Campbell RG, Gallagher PG, Kuttner JA (2001a) Fluid balance during and after an Ironman triathlon. Clin J Sport Med 11:44-50

Speedy DB, Noakes TD, Schneider C (2001b) Exercise-associated hyponatremia: a review. Emerg Med (Fremantle) 13:17-27

Speedy DB, Thompson JM, Rodgers I, Collins M, Sharwood K, Noakes TD (2002) Oral salt supplementation during ultradistance exercise. Clin J Sport Med 12:279-284

Stewart AD, Hannan WJ (2000) Prediction of fat mass and fat-free mass in male athletes using dual X-ray absorptiometry as the reference method. J Sports Sci 18:263-274
Stuempfle KJ, Lehmann DR, Case HS, Bailey S, Hughes SL, McKenzie J, Evans D (2002) Hyponatremia in a cold weather ultraendurance race. Alaska Med 44:51-55

Stuempfle KJ, Lehmann DR, Case HS, Hughes SL, Evans D (2003) Change in serum sodium concentration during a cold weather ultradistance race. Clin J Sport Med 13:171-175

Van Beaumont W (1972) Evaluation of hemoconcentration from hematocrit measurements. J Appl Physiol 32:712-713

Wharam PC, Speedy DB, Noakes TD, Thompson JM, Reid SA, Holtzhausen LM (2006) NSAID use increases the risk of developing hyponatremia during an Ironman triathlon. Med Sci Sports Exerc 38:618-622

Winger JM, Dugas JP, Dugas LR (2011) Beliefs about hydration and physiology drive drinking behaviours in runners. Br J Sports Med, epub ahead of print 